\title{
The contact binary V344 Lacertae: Is it a triple system?
}

\author{
Liu Liang ${ }^{1,2,3,4}$, Qian Shengbang ${ }^{1,2,3,4}$, Li Kai ${ }^{5}$, He Jiajia ${ }^{1,2,3}$, Li Linjia ${ }^{1,2,3}$, Zhao Ergang ${ }^{1,2,3}$ \\ and Li Xuzhi $1,2,3,4$
}

Received __; accepted _

Not to appear in Nonlearned J., 45.

\footnotetext{
${ }^{1}$ Yunnan Observatories, Chinese Academy of Sciences, 396 Yangfangwang, Guandu District, Kunming, 650216, P. R. China (e-mail: LiuL@ynao.ac.cn)

${ }^{2}$ Key Laboratory for the Structure and Evolution of Celestial Objects, Chinese Academy of Sciences, 396 Yangfangwang, Guandu District, Kunming, 650216, P. R. China

${ }^{3}$ Center for Astronomical Mega-Science, Chinese Academy of Sciences, 20A Datun Road, Chaoyang District, Beijing, 100012, P. R. China

${ }^{4}$ University of Chinese Academy of Sciences, Yuquan Road 19\#, Sijingshang Block, 100049 Beijing, China

${ }^{5}$ Shandong Provincial Key Laboratory of Optical Astronomy and Solar-Terrestrial Environment, Institute of Space Sciences, Shandong University, Weihai, 264209, China
} 


\begin{abstract}
The VRI passbands light curves of V344 Lac were presented and analyzed by using the latest version of the W-D code. The observed spectrum reveals that V344 Lac is not an A3 type but would be a later F type star according to the yielded temperature. The results of solution show that V344 Lac is an Asubtype contact binary, with a mediate photometric mass ratio of $0.387 \pm 0.003$ and a mediate contact factor of $44.6 \pm 3.0 \%$. Based on the parallax given by Gaia, the parameters of the components are estimated as: $M_{1}=1.16 \mathrm{M}_{\odot}, M_{2}=$ $0.45 \mathrm{M}_{\odot}, R_{1}=1.31 \mathrm{R}_{\odot}, R_{2}=0.88 \mathrm{R}_{\odot}, L_{1}=2.512 \mathrm{~L}_{\odot}, L_{2}=1.057 \mathrm{~L}_{\odot}$. The period investigation indicates that V344 Lac may have an eccentric orbital oscillation, with $P_{3}=12.4 \pm 0.5 \mathrm{yr}, A_{3}=0.0020 \pm 0.0002 \mathrm{~d}$, and $e=0.38 \pm 0.16$. Analysis shows such oscillation would be caused by a magnetic activity which can be explained by the Applegate mechanism. Meanwhile, according to the value of $l_{3}$ and the estimated physical parameters of V344 Lac, the mass of the third companion may be $0.79 M_{\odot}$. This third body could be a wide company.
\end{abstract}

Subject headings: Stars: binaries: close - Stars: binaries: eclipsing - Stars: individuals (V344 Lacertae) - Stars: evolution 


\section{Introduction}

Contact binaries are binary stars where both components have already overflowed their Roche lobe so that they have formed a common radiative or convective envelope with very strong interactions (e.g. Qian et al. 2017). Because of the contact in physical, they have the lowest angular momentum resulting from the tightest orbits among the same spectral type binaries, and they have very complex interactions. For example, the long-term cyclic behaviors of the orbital period would extend the life of contact binaries. A famous representative is the Thermal Relaxation Oscillations (TRO, Lucy 1976; Flannery 1976; Robertson \& Eggleton 1977), and the other possibility is the Common Convective Envelope Oscillations (CCEO, Liu et al. 2018). Hence, there are still unsolved problems about the evolution of contact binaries.

That contact binaries are formed from closed detached binaries is widely accepted now. The migration of angular momentum between the central pair and the third companion may be a very important process for the formation of a contact binary (e.g., Eggleton 2012). There are two reasons. First, contact binaries have been found in the young open cluster (e.g. TX Cnc, Liu et al. 2007; Zhang et al. 2009), indicating that formational time-scale of contact binaries could be much shorter than the time-scale of their nuclear evolution. Second, third bodies have been found in many contact binary systems. Pribulla \& Rucinski (2006) obtained $59 \pm 8 \%$ of contact binaries which are brighter than the magnitude of 10 and are in the northern-sky have a third companion. Rucinski et al. (2007) have tried to prove that the contact binaries with $P<1$ usually have an additional component by using of the adaptive optics technique. There are also some closed third bodies which were found by the combination of the $O-C$ method and the $l_{3}$ analysis from light curves (e.g., V345 Gem, Yang et al. 2009; MQ UMa, Zhou et al.|2015; V776 Cas, Zhou et al.|2016; V1005 Her, Zhu et al. 2019). 
V344 Lacertae was discovered as an EW-type binary by Miller \& Wachmann (1973) in a visual variable survey. After its discovery, except for minima monitoring, the only one of the published light curve analysis was given by Pribulla et al. (2005a), who yielded geometric elements of V344 Lacertae as $i=77.5(2), q=0.24, f=0.45(1)$. As Molik \& Wolf (2004) pointed out, V344 Lacertae is a special contact binary which is located in the blue envelope of the period-color diagram (e.g. Eggen 1967). However, after a spectroscopic observation, we found the spectral type of V344 Lac should be much later than A3-type. It may be not a contradictory with the period-color relationship.

In the present work, new photometric and spectroscopic data were presented in Section 2. Orbital period studies and light-curve analysis were given in Sections 3 and 4. Physical parameters of each component were estimated based on the Gaia parallax in Sections 5. In the last section, we interpreted the period variation.

\section{New photometric and spectroscopic data}

V344 Lacertae, for the purpose of orbital period investigation, had been observed from 2009 to 2014 by the 1-m and 60-cm reflecting telescopes at the Yunnan Observatories (YNOs), and by the 85-cm reflecting telescope at the Xinglong Station, the National Astronomical Observatory (NAO). The 1-m and 60-cm telescopes were equipped with the same Andor DW436 CCD cameras, combining a standard Johnson-Cousins filter system. The 85-cm telescope was equipped with a primary-focus multi-color CCD photometer where a PI1024 BFT (Back-illuminated and Frame-Transfer) camera was used (Zhou et al. 2009), generating an effective field of view $(\mathrm{FOV})$ as $16 .^{\prime} 5 \times 16 .^{\prime} 5$. And then on the nights of September 22, October 5 and 6, 2014, we obtained the three-color complete light curves (in $V R I$ filters) by the 1-m reflecting telescopes at the Weihai Observatory (WHO) of Shandong University, which was equipped a back-illuminated PIXIS 2048B CCD camera, with an 
FOV of $12^{\prime} 0 \times 12^{\prime} 0(\mathrm{Hu}$ et al. 2014). In all data reduction, 2MASS $22184267+5157291$ and 2MASS 22181951+5156086 were chosen as comparison and check star, respectively. The aperture photometry package PHOT which is for measuring magnitudes for a list of stars in IRAF was used to reduce the observed images, after a standard flat-fielding correction process. Finally, the complete $V R I$ light curves and the others partly light curves for minima were obtained. The original data with the standard errors of the complete light curves can be found in our electronic table. Phases were calculated with the equation $2456923.06759+0 .{ }^{d} 39224141 \times E$, where 2456923.06759 is the primary times of minimum obtained by the 1-m WHOT, $0 .{ }^{d} 39224141$ is our corrected linear orbital period (section 3), and $E$ is the epoch number. The corresponding light curves are shown in Figure 1. Several times of minima obtained by us with the parabolic fitting method are listed in Table 1 .

To determine the accurate temperature of V344 Lac, spectroscopic observations were obtained with the Yunnan Faint Object Spectrograph and Camera (YFOSC) attached to the 2.4-m telescope in Lijiang Gaomeigu Station on December 13, 2018. Grism-14 of which the bandpass is from 320 to $750 \mathrm{~nm}$ with a long-slit width of $2^{\prime \prime} .5$ was used (Fan et al. 2015). The exposure time was $600 \mathrm{~s}$. Bias subtraction and flat-field correction were made with standard IRAF procedures. The APEXTRACT package of the IRAF was used to extract the spectra. Wavelength calibration was performed using a He-Ne comparison lamp taken during the same observing run. University of Lyon Spectroscopic analysis Software (ULySS) package (Koleva et al. 2009; Wu et al. 2011) was used to analyze the spectra. The basic stellar atmospheric parameters were modeled as $T_{\text {eff }}=6312 \pm 54 \mathrm{~K}$ and the metallicity $[F e / H=-0.289 \pm 0.011]$. This value of temperature is similar to the result of Gaia (Gaia Collaboration et al. 2018). The corresponding spectral fitting is shown in the Figure 2. The fitting of the spectral at the larger wavelength region is not good. It may be account for the effect of the cooler component/components, because the subsequent analysis will reveal that there may be a cool third company in the system. 


\section{Orbital period analysis}

From 1948, the minima monitoring for V344 Lacertae has been over 70 years, which is helpful to study its long-term period variation. However, the data obtained by the visual or photograph method showed a very large scatter, so we discard those data in our analysis. Finally, 95 CCD or photoelectric times of minima of V344 Lac were collected and are listed in Table 2, including 10 of ours. These data were used to correct the linear ephemeris starting with the formula of $2456923.06759(17)+0 .{ }^{d} 39222768 \times E$. $T_{0}$ was one of our obtained minimum, and $P_{0}$ was obtained from Safar \& Zejda (2002) and Kholopov et al. (1985). The corrected new linear ephemeris is,

$$
\text { Min. } I=2456923.0683( \pm 0.0005)+0 .{ }^{d} 39224141( \pm 0.00000005) \times E
$$

The $(O-C)$ values with respect to the linear ephemeris are listed in the sixth column of Table 2. The corresponding $(O-C)$ diagram is displayed in Figure 3. The general $(O-C)$ trend of V344 Lac shown in Figure 3. In this figure, a possible cyclic variation is shown. We suppose that the variations in the $(O-C)$ diagrams are caused by the light-travel-time effect (LTTE) of a company orbiting in the binary system of elliptical orbit, then

$$
\begin{aligned}
(O-C)= & \Delta T+\Delta P \cdot E+\frac{\beta}{2} E^{2} \\
& +A_{3}\left[\left(1-e^{2}\right) \frac{\sin (\nu+\omega)}{1+e \cos \nu}+e \sin \omega\right]
\end{aligned}
$$

This formula was given by Irwin $(1952,1959)$, where $A_{3}=\left(a_{12} \sin i^{\prime}\right) / c$. The LevenbergMarquardt method was used in the non-linear fitting. The fitted parameters are listed in Table 3, while Equation (2) is plotted as a solid line in the upper panel of Figure 3. The final residuals of $(O-C)$ are tabulated in the seventh column of Table 2 and are shown in the lower panel of Figure 3 . 


\section{Photometric solutions of V344 Lac}

To determine photometric elements and further to understand the geometrical structure and evolutionary state of V344 Lac, the 2015 Version of the W-D code (Wilson \& Devinney 1971; Wilson 1979, 1990, 2008, 2012; van Hamme \& Wilson|2007; Wilson et al.|2010; Wilson \& van Hamme 2014) was used to analyze the obtained light curves. The $q$-search method was employed to find an initial value of $q$. We fixed $q$ at a series of values of $0.1,0.2,0.3$, etc., then fitted the light curves with the W-D code for each $q$ value, obtaining a series of corresponding fitting residuals, shown in Figure 4, and selected the value of $q$ with minimal residuals as the input parameter. The mass ratio thus should be wide range from 0.2 to 0.6 under each model.

During the solution, the bolometric albedo $A_{1}=A_{2}=0.5$ with the gravity-darkening coefficient of $g_{1}=g_{2}=0.32$ for convective equilibrium are used (Lucy 1967; Rucinski 1969), respectively. According to Claret \& Gimenez (1990), the square root limb-darkening coefficients were used. We adjusted the mass ratio $q$; the mean temperature of star 2 , $T_{2}$; the monochromatic luminosity of star 1 and the dimensionless potential of star 1 . We started the DC program at model 2. After thousands of iterations it converged to model 3 $\left(\Omega_{1}=\Omega_{2}\right.$, mode 3 for contact configuration). Because the $(O-C)$ diagram presented some cyclic variation, we ran the program with the adjusted parameter of $l_{3}$ light. The

photometric solutions are listed in Table 4 and the theoretical light curves computed with those photometric elements are plotted in Figure 1, while the geometric constructions of V344 Lac are shown in Figure 5. 


\section{Estimation of the physical parameters with the Gaia parallax}

Physical parameters such as mass, radius and luminosity are very important information for a contact binary system. Hence it is necessary to estimate them. Here, we will introduce how to estimate the physical parameters without radial velocity curves.

We have known that the Parallax of V344 Lac is $1.6266 \pm 0.0363$ mas (Gaia DR2 Gaia Collaboration et al. 2018). According to the formula of

$$
(m-M)_{V}=10-5 \lg \text { para }
$$

we obtain a distance modulus of $8.944 \pm 0.049 \mathrm{mag}$. The V-band magnitude of the comparison star (2MASS 22184267+5157291) is 13.792 mag (URAT1 Catalog, Zacharias et al. 2015). According to our V-band light curve (Figure 1), the magnitude of V344 Lac should be from 12.299 to 12.697 mags in the V-band. The composite V-band absolute magnitude therefore is about 3.355 mags. Adopted a bolometric correction (e.g., $\mathrm{BCv}$ $=-0.027 \mathrm{mag}$ for $T=6312 \mathrm{~K}$, Worthey \& Lee 2011), the absolute bolometric magnitude of V344 Lac should be 3.328 mags. After a bolometric correction, the bolometric absolute magnitude and the total luminosity are obtained with the relationships

$$
\begin{gathered}
M_{\mathrm{bol}}=M_{V}+\mathrm{BCv} \\
L_{T}=L_{1}+L_{2}+L_{3}=10^{-0.4\left(M_{\mathrm{bol}}-4.83\right)} \cdot \mathrm{L}_{\odot} .
\end{gathered}
$$

$\mathrm{BCv}$ is $-0.027 \mathrm{mag}$ for the temperature of $6312 \mathrm{~K}$ (Worthey \& Lee 2011). The total luminosity obtained in this calculation is independent of the photometric solution. According to the law of blackbody, we have

$$
L_{1}+L_{2}=\left(\frac{T_{1}}{T_{\odot}}\right)^{4}\left(A r_{1}\right)^{2}+\left(\frac{T_{2}}{T_{\odot}}\right)^{4}\left(A r_{2}\right)^{2},
$$

where $T_{\odot}$ is the effective temperature of the Sun (adopting $5770 \mathrm{~K}$ ); $A$ is the separation of the two components; $r_{1}$ and $r_{2}$ are the relative radii for star 1 and star 2, respectively 
$\left(r_{i}=\left(r_{i \text { pole }} \cdot r_{i \text { side }} \cdot r_{i \text { back }}\right)^{1 / 3}\right)$. Combine Eq 5 and 6 , we obtain $A$ as follows,

$$
A=\left[10^{-0.4\left(M_{\mathrm{bol}}-4.83\right)}-L_{3}\right]^{\frac{1}{2}}\left[\left(\frac{T_{1}}{T_{\odot}}\right)^{4} r_{1}^{2}+\left(\frac{T_{2}}{T_{\odot}}\right)^{4} r_{2}^{2}\right]^{-\frac{1}{2}} .
$$

The contribution of $l_{3}$ are known from the photometric solution in Section 4 . Then, by using of the Kepler's third law,

$$
\frac{A^{3}}{P^{2}}=74.5\left(M_{1}+M_{2}\right)
$$

where $A$ is in unit of solar radius, while $P$ is in days, and $M_{1}, M_{2}$ are in solar mass. The total mass therefore can be determined. Because the mass ratio $q=M_{2} / M_{1}$ has been obtained, the individual mass of component is also known. Hence, the absolute physical parameters are estimated as follows, $M_{1}=1.16 \mathrm{M}_{\odot}, M_{2}=0.45 \mathrm{M}_{\odot}, R_{1}=1.31 \mathrm{R}_{\odot}$, $R_{2}=0.88 \mathrm{R}_{\odot}, L_{1}=2.512 \mathrm{~L}_{\odot}, L_{2}=1.057 \mathrm{~L}_{\odot}$.

Uncertainties of this estimation can be given by the error transfer formula,

$$
\Delta M_{\mathrm{bol}}=\Delta m_{V}+\frac{5}{\ln 10} \frac{\Delta \text { para }}{\text { para }}+\Delta \mathrm{BCv}
$$

and

$$
\frac{\Delta M}{M_{\text {tot }}}=\frac{3}{2} \frac{\Delta L_{T}+\Delta L_{3}}{L_{T}-L_{3}}+\frac{6 r_{1}^{2} T_{1}^{3} \Delta T_{1}+3 T_{1}^{4} r_{1} \Delta r_{1}+6 r_{2}^{2} T_{2}^{3} \Delta T_{2}+3 T_{2}^{4} r_{2} \Delta r_{2}}{T_{1}^{4} r_{1}^{2}+T_{2}^{4} r_{2}^{2}}+\frac{2 \Delta P}{P} .
$$

Having ignored the high-order-small-quantities, we have

$$
\frac{\Delta M}{M_{\mathrm{tot}}} \simeq \frac{0.6 \ln 10 \cdot L_{T} \Delta M_{\mathrm{bol}}+1.5 \Delta L_{3}}{L_{T}-L_{3}}+\frac{6 r_{1}^{2} T_{1}^{3} \Delta T_{1}+6 r_{2}^{2} T_{2}^{3} \Delta T_{2}}{T_{1}^{4} r_{1}^{2}+T_{2}^{4} r_{2}^{2}}
$$

In most situations, $\Delta T / T<0.01$. Hence, for a safe estimation, $\Delta M / M_{\text {tot }} \simeq 0.14$ when $\Delta M_{\mathrm{bol}}=0.1 \mathrm{mag}$.

\section{Discussion and conclusions}

By the solution of the multiple color light curves, we found that V344 Lac is a moderate mass ratio $(0.387 \pm 0.003)$, moderate contact binary $(44.6 \pm 3.0 \%)$. The more massive 
component is the hotter one. Hence it is an A-subtype contact binary system (Binnendijk 1970). Combined with the parallax given by Gaia DR2, the physical parameters of each component are estimated.

Based on all available photoelectric and CCD eclipsing times, the possible period changes of V344 Lac were analyzed in Section 3. The orbital period was revised as 0.39224141 day by using the 95 timings being listed in Table 2. The orbital period shows an eccentric cyclic variation (Figure 3). Because both the primary and the secondary times of light minimum follow the same general trend of $(O-C)$ variation, it is excluded that the oscillation was caused by the apsidal motions. Cyclic period change can be caused by the mechanism of magnetic activity (e.g., Applegate 1992; Lanza et al. 1998). Figure 6 shows the required luminosity to drive the Applegate mechanism. It is a very small fraction of the luminosity for both components. Hence the Applegate mechanism could be an explanation of the period oscillation. There was not an obvious cool spot in our observed light curves could be on account of the halcyon period according to location of time in the $(O-C)$ diagram. On the other hand, because of the solution of $l_{3}$, the LTTE due to the presence of a third body (e.g., Liao \& Qian 2010) is also discussed here. With the equation of

$$
f(m)=\frac{\left(m_{3} \sin i^{\prime}\right)^{3}}{\left(M_{1}+M_{2}+m_{3}\right)^{2}},
$$

it is obtained that the minima mass (at $i^{\prime}=90^{\circ}$ ) of the third body is estimated to be $m_{3 \min }=0.10 \pm 0.05 M_{\odot}$, and that an orbital semimajor axis shorter than $6.40 \pm 0.61 \mathrm{au}$. However, according to the solution and the estimated physical parameters of V344 Lac, $L_{3}$ could be $0.397 \mathrm{~L}_{\odot}$. The mass of a main-sequence star with such luminosity is about 0.79 solar mass, corresponding to a K0 type star (Cox 2000). The mass of the third body will reach this value only while $i^{\prime}$ is very small.

Some well-studied contact binaries which have cyclic oscillation in their period are collected in Table 5. The eleventh column denotes detection of cool spot in their light 
curves. In this table, we find that most of the oscillations are non-eccentric, and 12 of the 35 were shown $l_{3}$ in their photometric solutions. Cool spots and third light have been found in the same time in five samples. Especially, V776 Cas has been found a third light both by the spectroscopic method (D'Angelo et al. 2006) and the photometric solution (Zhou et al. 2016). However, the third body of V776 Cas may be a wide company (The separation is about 5.38 arcsec, D'Angelo et al. 2006). Hence, for V344 Lac, the $l_{3}$ in photometric solution may be on account of a wide K0 type third body, while the oscillation in orbital period should be due to a 12.4-years-magnetic-activity.

The third body in a contact binary system is a common phenomenon. Several studies suggest that a similarly large fraction of contact binaries have a third component Pribulla \& Rucinski 2006; D'Angelo et al. 2006; Rucinski et al. 2007); even the fraction is as large as 96\% (Tokovinin et al. 2006). The probability of a third body in V344 Lac is therefore high.

The third body also would effect the formation of V344 Lac. Fabrycky \& Tremaine (2007) investigated the orbital shrinkage by Kozai effect (Kozai 1962) and tidal friction, concluding that recently binary stars with orbital periods of $0.1 \sim 10$ days are produced from binaries with much longer periods $\left(10 \sim 10^{5}\right.$ days $)$ via the presented tertiaries. The same conclusion was obtained by Qian et al. (2018) via a statistic work for EA-type binaries. On the other hand, Qian et al. (2013a, b, 2014b) had emphasized for many times that the relative close companion played an important role by exchanging angular momentum between the binary and the companion, which reduces angular momentum of central close binaries then forming a compact initial orbit. V344 Lac may have been formed in this way, too.

We are grateful to the anonymous referee who has given very useful suggestions to improve the paper. This work is partly supported by the Chinese Natural Science Foundation (No.11773066), the young academic and technology leaders project of Yunnan 
Province (No. 2015HB098), and the Open Project Program of the Key Laboratory of Optical Astronomy, National Astronomical Observatories, Chinese Academy of Sciences. New observations of the systems were obtained with the 2.4-m telescope of Lijiang station of Yunnan Observatories, the 1-m telescope at the Weihai Observatory (WHO) of Shandong University, with the 85-cm telescope at Xinglong observation base, and with the 1-m and 60-cm telescope at Yunnan Observatories (YNOs). We would like to thank all the staff of the 2.4-m telescope of Lijiang station of Yunnan Observatories for their help and support during our observations. We also thank the persons who observed or reduced the data. 


\section{REFERENCES}

Agerer, F., Hubscher, J., 2003, IBVS No. 5484, 1

Applegate J. H. 1992, ApJ, 385, 621

Binnendijk, L. 1970, Vistas Astron., 12, 217

Brat, L., Zejda, M., Svoboda, P. 2007, OEJV No. 74, 1

Claret, A., and Gimenez, A., 1990, A\&A 230, 412

Cox, A. N. 2000, Allen's Astrophysical Quantities (4th ed., NewYork: Springer)

Dai, H.-F., Yang, Y.-G., \& Yin, X.-G. 2011, NewA, 16, 173

D’Angelo, C., van Kerkwijk, M. H., Rucinski, S. M. 2006, AJ, 132, 650

Diethelm, R. 2003 IBVS No. 5438, 1

Diethelm, R. 2005, IBVS No. 5653, 1

Diethelm, R. 2012, IBVS No. 6011, 1

Dvorak, S. W. 2003, IBVS No. 5378, 1

Dvorak, S. W. 2006, IBVS No. 5677, 1

Eggen, O. J. 1967, MemRAS, 70, 111

Eggleton, P. P. 2012, JASS, 29, 145

Fabrycky, D. \& Tremaine, S. 2007, AJ, 669, 1298

Fan Y. F., Bai J. M., Zhang J. J., Wang C. J., Chang L., Xin Y. X., Zhang R. L., 2015, Res. Astron. Astrophys., 15, 918 
Flannery, B. P. 1976, ApJ, 205, 217

Gaia Collaboration, Brown, A. G. A., Vallenari, A et al. 2018, A\&A, 616A, 1

He, J.-J. \& Qian, S.-B. 2010, PASJ, 62, 441

Hoffmann, M. 1983, IBVS No. 2344, 1

Hu Shao-Ming, Han Sheng-Hao, Guo Di-Fu and Du Jun-Ju 2014, RAA, Vol. 14 No. 6, $719 \mathrm{C} 732$

Hubscher, J., Lehmann, P. B. 2012, IBVS No. 6026, 1

Hubscher, J., Paschke, A., Walter, F. 2005, IBVS No. 5657, 1

Hubscher, J., Paschke, A., Walter, F. 2006, IBVS No. 5731, 1

Hubscher, J., Steinbach, H.-M., Walter, F. 2009, IBVS No. 5874, 1

Hubscher, J., Lehmann, P. B., Monninger, G., Steinbach, H.-M., Walter, F. 2010, IBVS No. 5941,1

Hubscher, J., Lehmann, P. B., Walter, F. 2012, IBVS No. 6010, 1

Hubscher, J., 2005, IBVS No. 5643, 1

Hubscher, J. 2011, IBVS No. 5984, 1

Hubscher, J. 2015, IBVS No. 6152, 1

Irwin, J. B. 1952, ApJ, 116, 211

Irwin, J. B. 1959, ApJ, 64, 149

Kholopov, P. N., Samus, N. N., Kazarovets, E. V., Perova, N. B. 1985, IBVS No. 2681, 1 
Koleva, M., Prugniel, Ph., Bouchard, A., Wu, Y. 2009, A\&A, 501, 1269

Kopal, D.: Dynamics of Close Binary Systems. D. Reidel, Dordrecht(1978)

Kotkova, L., Wolf, M. 2006, IBVS No. 5676, 1

Kozai, Y. 1962, AJ, 67, 591

Lanza A. F., Rodono M. Rosner R., 1998, MNRAS, 296, 893

Lee, J. W., Youn, J.-H., Han, W.-Y., et al. 2010, AJ, 139, 898

Li, K., Qian, S. -B., Hu, S. -M. \& He, J. -J.

Li, K., Hu, S. -M., Guo, D. -F., Jiang, Y. -G., Gao, Y. -G., Chen, X. \& Odell, Andrew P 2015, AJ, 149, 120L

Liao, W.-P. \& Qian, S.-B. 2010, MNRAS, 405, 1930

Liao, W. -P., Qian, S. -B. \& Liu, N. -P. 2012, AJ, 144, 178L

Liao, W. -P., Qian, S. -B., Li, K., He, J. -J., Zhao, E. -G. \& Zhou, X. 2013, AJ, 146, 79L

Liao, W. -P., Qian, S. -B., Zhao, E. -G. \& Jiang, L. -Q. 2014, NewA, 31, 65L

Liao, W. -P., Qian, S. -B. \& Sarotsakulchai, T. 2019, AJ, 157, 207L

Liao, W. -P. \& Sarotsakulchai, T. 2019, PASP, 131a, 4202L

Liu, Liang, Qian, Sheng-Bang, Boonrucksar, Soonthornthum, Zhu, Li-Ying, He, Jia-Jia and Yuan, J. -Z. 2007, PASJ, 59, 607

Liu, L., Qian, S. B., He, J.-J., Zhang, J. \& Li, L.-J. 2009, Ap\&SS, 321, 19

Liu, L., Qian, S. -B., Liao, W. -P., He, J. -J., Zhu, L. -Y., Li, L. -J. \& Zhao, E. -G. 2011, AJ, 141, 44L 
Liu, Liang, Qian, Shengbang, He, Jiajia, Liao, Wenping \& Liu, Nianping 2016, PASJ, 68, $31 \mathrm{~L}$

Liu, L., Qian, S. B. \& Xiong, X. 2018, MNRAS, 474, 5199

Liu, N.-P., Qian, S.-B., Wang, J.-J., et al. 2014, NewA, 32, 31

Lu, Hongpeng, Zhang, Liyun, Han, Xianming L., Pi, Qingfeng \& Wang, Daimei 2016, NewA, 48, 58L

Lucy, L. B. 1967, Zeitschrift für Astrophysik, 65, 89

Lucy, L. B. 1976, ApJ, 205, 208

Miller, W. J. \& Wachmann, A. A. 1973, RA, 8, 367

Molik P. \& Wolf, M. 2004, BaltA, 13, 145

Parimucha, S., Vanko, M., Pribulla, T. et al. 2007, IBVS No. 5777, 1

Parimucha, S., Dubovsky, P., Kudak, V., Perig, V. 2016, IBVS No. 6167, 1

Prasad, Vinod, Pandey, J. C., Patel, M. K. \& Srivastava, D. C. 2014, Ap\&SS, 353, 575P

Pribulla, T. \& Rucinski, S. M. 2006, AJ, 131, 2986

Pribulla, T., Vanko, M., Chochol, D., Parimucha, S., Baludansky, D. 2005, Ap\&SS, 296, 281

Pribulla, T., Baludansky, D., Chochol, D. et al. 2005, IBVS No. 5668, 1

Qian, S.-B., Zhu, L.-Y., Soonthornthum, B. 2005, NewA, 11, 52

Qian, S.-B., He, J.-J. \& Xiang, F.-Y. 2008, PASJ, 60, 77 
Qian, S. -B., Li, K., Liao, W. -P., Liu, L., Zhu, L. -Y., He, J. -J., Wang, J. -J. \& Zhao, E. -G. 2013a, AJ, 145, 91Q

Qian, S.-B., Liu, N.-P., Li, K., et al. 2013b, ApJS, 209, 13

Qian, S.-B., Zhang, J., Wang, J.-J., et al. 2013c, ApJS, 207, 22

Qian, S.-B., Wang, J.-J., Zhu, L.-Y., et al. 2014b, ApJS, 212, 4

Qian, S. -B., Zhou, X., Zola, S., Zhu, L. -Y., Zhao, E. -G., Liao, W. -P. \& Leung, K. -C. 2014a, AJ, 148, 79Q

Qian, S.-B., He, J.-J., Zhang, J., et al. 2017, RAA, 17, 87

Qian, S.-B., Zhang, J., He, J.-J., Zhu, L.-Y., Zhao, E.-G., Shi, X.-D., Zhou, X., Han, Z.-T. 2018, ApJS, 235, 5

Robertson, J. A. \& Eggleton, P. P. 1977, MNRAS, 179, 359

Rucinski, S. M., Pribulla, T., van K. M. H. 2007, AJ, 134, 2353

Rucinski, S. M. 1969, AcA, 19, 245

Safar, J., Zejda, M. 2000, IBVS No. 4887, 1

Safar, J., Zejda, M. 2002, IBVS No. 5263, 1

Tokovinin, A., Thomas, S., Sterzik, M., Udry, S. 2006, A\&A, 450, 681

Tout, C. A., Hall, D. S. 1991, MNRAS, 253, 9T

Van Hamme, W., \& Wilson, R. E. 2007, ApJ, 661, 1129

Wang, J.-J., Qian, S.-B., He, J.-J., Li, L. J. \& Zhao, E. G. 2014, AJ, 148, 95

Williams, S. C., Darnley, M. J., Bode, M. F., Steele, I. A. 2015, ApJ, 805L, 18 
Wilson, R. E., \& Devinney, E. J. 1971, ApJ, 166, 605

Wilson, R. E., \& van Hamme, W. 2014, ApJ, 780, 151

Wilson, R. E., Van Hamme, W., \& Terrell, D. 2010, ApJ, 723, 1469

Wilson, R. E. 1979, ApJ, 234, 1054

Wilson, R. E. 1990, ApJ, 356, 613

Wilson, R. E. 2008, ApJ, 672, 575

Wilson, R. E. 2012, AJ, 144, 73

Worthey, G., \& Lee, H.-C. 2011, ApJS, 193, 1

Wu, Yue, Singh, H. P., Prugniel, P., Gupta, R., Koleva, M. 2011, A\&A, 525A, 71

Xiang, F. -Y., Xiao, T. -Y., Zhang, B. \& Shi, X. -D. 2015a, AJ, 150, 9X

Xiang, Fu-Yuan, Xiao, Ting-Yu \& Yu, Yun-Xia 2015b, AJ, 150, 25X

Xiang, Fu-Yuan, Yu, Yun-Xia \& Xiao, Ting-Yu 2015c, AJ, 149, 62X

Yang, Y.-G., Qian, S.-B. \& Zhu, C.-H. 2004, PASP, 116, 826

Yang, Y.-G., Qian, S.-B., Zhu, L.-Y., et al. 2008, PASJ, 60, 803

Yang, Y.-G., Qian, S.-B., Zhu, L.-Y. and He, J.-J. 2009, AJ, 138, 540

Yang, Y.-G., Li, H.-L. \& Dai, H.-F. 2013, AJ, 145, 60

Zacharias, N., Finch, C., Subasavage, J., et al. 2015, AJ, 150, 101

Zasche, P., Liakos, A., Niarchos, P., et al. 2009, NewA, 14, 121

Zejda, M., Mikulasek, Z., Wolf, M. 2006, IBVS No. 5741, 1 
Zejda, M. 2004, IBVS No. 5583, 1

Zhang, X.-B., Deng, L. and Lu, P. 2009, AJ, 138, 680

Zhou A.-Y., Jiang X.-J., Zhang Y.-P., Wei J.-Y. 2009, RAA, 9, 349

Zhou, X., Qian, S.-B., Liao, W.-P., Zhao, E.-G., Wang, J.-J. and Jiang, L.-Q. 2015, AJ, 150,83

Zhou, X., Qian, S.-B., Zhang, J., Jiang, L.-Q., Zhang, B. and Kriener, J. 2016, ApJ, 817, 133

Zhu, L. Y., Qian, S. B., Liu, N. P., Liu, L. \& Jiang, L. Q.

Zhu, L.-Y., Wang, Z.-H., Tian, X.-M., Li, L.-J. and Gao, X. 2019, MNRAS, 489, 2677 
Table 1: New times of light minima for V344 Lac.

\begin{tabular}{lllll}
\hline J.D. (Hel.) (d) & Error (d) & Min. & Filter & Telescope \\
\hline 2455071.29617 & 0.00033 & $\mathrm{~s}$ & $R$ & XL $85 \mathrm{~cm}$ \\
2455132.09126 & 0.00012 & $\mathrm{~s}$ & $R$ & YNO $60 \mathrm{~cm}$ \\
2455492.16933 & 0.00080 & $\mathrm{~s}$ & $R$ & YNO $60 \mathrm{~cm}$ \\
2455501.19018 & 0.00025 & $\mathrm{~s}$ & $R$ & YNO $60 \mathrm{~cm}$ \\
2455535.11975 & 0.00063 & $\mathrm{p}$ & $R$ & YNO $60 \mathrm{~cm}$ \\
2455816.15762 & 0.00050 & $\mathrm{~s}$ & $R$ & YNO $60 \mathrm{~cm}$ \\
2455876.17208 & 0.00047 & $\mathrm{~s}$ & $R$ & YNO $1 \mathrm{~m}$ \\
2456923.06757 & 0.00017 & $\mathrm{p}$ & $V$ & WHO $1 \mathrm{~m}$ \\
2456923.06757 & 0.00013 & $\mathrm{p}$ & $R$ & WHO $1 \mathrm{~m}$ \\
2456923.06762 & 0.00016 & $\mathrm{p}$ & $I$ & WHO $1 \mathrm{~m}$ \\
2456936.20572 & 0.00108 & $\mathrm{p}$ & $V$ & WHO $1 \mathrm{~m}$ \\
2456936.20833 & 0.00118 & $\mathrm{p}$ & $R$ & WHO $1 \mathrm{~m}$ \\
2456936.20945 & 0.00103 & $\mathrm{p}$ & $I$ & WHO $1 \mathrm{~m}$ \\
2456936.99921 & 0.00107 & $\mathrm{p}$ & $V$ & WHO $1 \mathrm{~m}$ \\
2456936.99631 & 0.00160 & $\mathrm{p}$ & $R$ & WHO $1 \mathrm{~m}$ \\
2456936.99553 & 0.00105 & $\mathrm{p}$ & $I$ & WHO $1 \mathrm{~m}$ \\
\hline
\end{tabular}

Note: "XL 85cm" refers to the 85cm telescope at the Xinglong Station, the National Astronomical Observatory. "YNO 60cm" and "YNO 1m" refer to the $60 \mathrm{~cm}$ and $1 \mathrm{~m}$ telescopes at the Yunnan Observatories, respectively, while "WHO $1 \mathrm{~m}$ " denotes the $1 \mathrm{~m}$ telescope at the Weihai Observatory (WHO) of Shandong University. 
Table 2. All available Times of light minima with errors for V344 Lac.

\begin{tabular}{|c|c|c|c|c|c|c|c|}
\hline JD (Hel.) & Filter & Min. & Error (d) & Epoch & $(O-C)(\mathrm{d})$ & Residuals (d) & Reference \\
\hline 2450658.3828 & & $\mathrm{~s}$ & 0.0014 & -15971.5 & -0.00177 & -0.00052 & IBVS No. 4887 \\
\hline 2451142.4126 & & $\mathrm{~s}$ & 0.0027 & -14737.5 & 0.00213 & 0.00453 & IBVS No. 5263 \\
\hline 2451467.3782 & V & $\mathrm{p}$ & 0.0017 & -13909 & -0.00428 & -0.00209 & IBVS No. 5263 \\
\hline 2451467.5729 & V & $\mathrm{s}$ & 0.0026 & -13908.5 & -0.00570 & -0.00351 & IBVS No. 5263 \\
\hline 2451782.5480 & & $\mathrm{~s}$ & 0.0028 & -13105.5 & -0.00049 & 0.00111 & OEJV No. 0074 \\
\hline 2451786.4683 & & $\mathrm{~s}$ & 0.0007 & -13095.5 & -0.00257 & -0.00098 & IBVS No. 5484 \\
\hline 2451806.4738 & & $\mathrm{~s}$ & 0.0002 & -13044.5 & -0.00138 & 0.00017 & IBVS No. 5484 \\
\hline 2451817.4552 & & s & 0.0009 & -13016.5 & -0.00274 & -0.00122 & IBVS No. 5484 \\
\hline 2451817.651 & & $\mathrm{p}$ & 0.005 & -13016 & -0.00306 & -0.00154 & IBVS No. 5484 \\
\hline 2452123.4064 & & $\mathrm{~s}$ & 0.0007 & -12236.5 & 0.00016 & 0.00099 & IBVS No. 5484 \\
\hline 2452134.3950 & & $\mathrm{~s}$ & 0.0022 & -12208.5 & 0.00600 & 0.00680 & IBVS No. 5484 \\
\hline 2452134.586 & & $\mathrm{p}$ & 0.003 & -12208 & 0.00088 & 0.00168 & IBVS No. 5484 \\
\hline 2452194.4006 & & $\mathrm{~s}$ & 0.0018 & -12055.5 & -0.00133 & -0.00067 & IBVS No. 5484 \\
\hline 2452194.5965 & & $\mathrm{p}$ & 0.0013 & -12055 & -0.00155 & -0.00089 & IBVS No. 5484 \\
\hline 2452228.3320 & & $\mathrm{p}$ & 0.0018 & -11969 & 0.00119 & 0.00178 & IBVS No. 5484 \\
\hline 2452228.5257 & & s & 0.0001 & -11968.5 & -0.00123 & -0.00064 & IBVS No. 5484 \\
\hline 2452505.4496 & & $\mathrm{~s}$ & 0.0007 & -11262.5 & 0.00023 & 0.00021 & IBVS No. 5484 \\
\hline 2452531.5346 & & $\mathrm{p}$ & 0.0010 & -11196 & 0.00118 & 0.00110 & IBVS No. 5438 \\
\hline 2452592.3311 & & $\mathrm{p}$ & 0.0010 & -11041 & 0.00021 & 0.00015 & IBVS No. 5438 \\
\hline 2452601.5480 & & $\mathrm{~s}$ & 0.0052 & -11017.5 & -0.00052 & -0.00074 & IBVS No. 5378 \\
\hline 2452621.3567 & & $\mathrm{p}$ & 0.0010 & -10967 & -0.00001 & -0.00027 & IBVS No. 5484 \\
\hline 2452872.3917 & $R$ & $\mathrm{p}$ & 0.0030 & -10327 & 0.00049 & -0.00024 & IBVS No. 5583 \\
\hline 2452872.3917 & V & $\mathrm{p}$ & 0.0029 & -10327 & 0.00049 & -0.00024 & IBVS No. 5583 \\
\hline 2452872.3921 & $I$ & $\mathrm{p}$ & 0.0029 & -10327 & 0.00089 & 0.00016 & IBVS No. 5583 \\
\hline 2452875.5229 & V & $\mathrm{p}$ & 0.0033 & -10319 & -0.00624 & -0.00698 & IBVS No. 5583 \\
\hline 2452875.5298 & $R$ & $\mathrm{p}$ & 0.0035 & -10319 & 0.00066 & 0.00102 & IBVS No. 5583 \\
\hline 2452875.5309 & $I$ & $\mathrm{p}$ & 0.0034 & -10319 & 0.00176 & 0.00037 & IBVS No. 5583 \\
\hline 2452878.4734 & & $\mathrm{~s}$ & 0.0006 & -10311.5 & 0.00245 & 0.00171 & IBVS No. 5643 \\
\hline 2452896.5148 & $B V R$ & $\mathrm{~s}$ & 0.0001 & -10265.5 & 0.00074 & -0.00003 & IBVS No. 5668 \\
\hline 2452901.4175 & $B V R$ & $\mathrm{p}$ & 0.0001 & -10253 & 0.00043 & -0.00036 & IBVS No. 5668 \\
\hline 2452901.6141 & $B V R$ & $\mathrm{~s}$ & 0.0002 & -10252.5 & 0.00091 & 0.00012 & IBVS No. 5668 \\
\hline 2452903.3793 & $B V R$ & $\mathrm{p}$ & 0.0001 & -10248 & 0.00102 & 0.00023 & IBVS No. 5668 \\
\hline 2452925.3448 & $R$ & $\mathrm{p}$ & 0.0001 & -10192 & 0.00100 & 0.00018 & IBVS No. 5676 \\
\hline 2452925.5409 & $R$ & $\mathrm{~s}$ & 0.0001 & -10191.5 & 0.00098 & 0.00016 & IBVS No. 5676 \\
\hline 2452941.4260 & & $\mathrm{p}$ & 0.0003 & -10151 & 0.00030 & -0.00055 & IBVS No. 5643 \\
\hline 2452955.3506 & $I$ & $\mathrm{~s}$ & 0.0003 & -10115.5 & 0.00033 & -0.00054 & IBVS No. 5668 \\
\hline 2452956.3321 & $I$ & $\mathrm{p}$ & 0.0003 & -10113 & 0.00123 & 0.00036 & IBVS No. 5668 \\
\hline 2452957.3119 & $I$ & $\mathrm{~s}$ & 0.0001 & -10110.5 & 0.00043 & -0.00045 & IBVS No. 5668 \\
\hline 2452957.5088 & I & $\mathrm{p}$ & 0.0002 & -10110 & 0.00121 & 0.00033 & IBVS No. 5668 \\
\hline 2453222.4672 & & $\mathrm{~s}$ & 0.001 & -9434.5 & 0.00053 & -0.00073 & IBVS No. 5657 \\
\hline 2453233.4496 & & $\mathrm{~s}$ & 0.0002 & -9406.5 & 0.00017 & -0.00110 & IBVS No. 5657 \\
\hline 2453242.4693 & & $\mathrm{~s}$ & 0.0024 & -9383.5 & -0.00168 & -0.00335 & IBVS No. 5657 \\
\hline 2453256.3940 & & $\mathrm{p}$ & 0.0005 & -9348 & -0.00155 & -0.00324 & IBVS No. 5657 \\
\hline 2453256.5921 & & $\mathrm{~s}$ & 0.0002 & -9347.5 & 0.00043 & -0.00087 & IBVS No. 5657 \\
\hline 2453259.3397 & $R_{c}$ & $\mathrm{~s}$ & 0.0007 & -9340.5 & 0.00234 & 0.00104 & IBVS No. 5741 \\
\hline
\end{tabular}


Table 2-Continued

\begin{tabular}{|c|c|c|c|c|c|c|c|}
\hline JD (Hel.) & Filter & Min. & Error (d) & Epoch & $(O-C)(\mathrm{d})$ & Residuals (d) & Reference \\
\hline 2453259.5344 & $R_{c}$ & $\mathrm{p}$ & 0.0004 & -9340 & 0.00092 & -0.00038 & IBVS No. 5741 \\
\hline 2453259.5359 & & $\mathrm{p}$ & 0.0042 & -9340 & 0.00242 & 0.00112 & IBVS No. 5657 \\
\hline 2453260.5157 & $R$ & $\mathrm{~s}$ & 0.0001 & -9337.5 & 0.00162 & 0.00031 & IBVS No. 5676 \\
\hline 2453284.4405 & & $\mathrm{~s}$ & 0.0065 & -9276.5 & -0.00031 & -0.00202 & IBVS No. 5657 \\
\hline 2453343.282 & & $\mathrm{~s}$ & 0.002 & -9126.5 & 0.00498 & -0.00164 & IBVS No. 5653 \\
\hline 2453637.4594 & & $\mathrm{~s}$ & 0.0003 & -8376.5 & 0.00132 & -0.00031 & IBVS No. 5731 \\
\hline 2453704.5331 & $V$ & s & 0.0003 & -8205.5 & 0.00174 & 0.00008 & IBVS No. 5677 \\
\hline 2453928.5027 & & $\mathrm{~s}$ & 0.0001 & -7634.5 & 0.00149 & -0.00019 & IBVS No. 5777 \\
\hline 2453932.4253 & & $\mathrm{~s}$ & 0.0008 & -7624.5 & 0.00168 & 0.00000 & IBVS No. 5731 \\
\hline 2453939.4857 & & $\mathrm{~s}$ & 0.0001 & -7606.5 & 0.00174 & 0.00006 & IBVS No. 5777 \\
\hline 2454004.4019 & & $\mathrm{p}$ & 0.0001 & -7441 & 0.00198 & 0.00032 & IBVS No. 5777 \\
\hline 2454018.5225 & & $\mathrm{p}$ & 0.0002 & -7405 & 0.00189 & 0.00023 & IBVS No. 5777 \\
\hline 2454019.5060 & & $\mathrm{~s}$ & 0.0004 & -7402.5 & 0.00477 & 0.00307 & OEJV No. 0074 \\
\hline 2454068.336 & & $\mathrm{p}$ & 0.0003 & -7278 & 0.00073 & -0.00090 & IBVS No. 5777 \\
\hline 2454453.3229 & & $\mathrm{~s}$ & 0.0003 & -6296.5 & 0.00269 & 0.00148 & IBVS No. 5874 \\
\hline 2455033.4456 & & $\mathrm{~s}$ & 0.0026 & -4817.5 & 0.00034 & 0.00075 & IBVS No. 5941 \\
\hline 2455051.4876 & & $\mathrm{~s}$ & 0.0003 & -4771.5 & -0.00076 & -0.00028 & IBVS No. 5941 \\
\hline 2455062.4707 & & $\mathrm{~s}$ & 0.0005 & -4743.5 & -0.00042 & 0.00010 & IBVS No. 5941 \\
\hline 2455071.29617 & $R$ & $\mathrm{p}$ & 0.00033 & -4721 & -0.00038 & 0.00017 & This paper \\
\hline 2455132.09126 & $R$ & $\mathrm{p}$ & 0.00012 & -4566 & -0.00271 & -0.00193 & This paper \\
\hline 2455141.3114 & & $\mathrm{~s}$ & 0.0009 & -4542.5 & -0.00025 & 0.00057 & IBVS No. 5941 \\
\hline 2455141.5065 & & $\mathrm{p}$ & 0.0003 & -4542 & -0.00127 & -0.00045 & IBVS No. 5941 \\
\hline 2455445.4929 & & $\mathrm{p}$ & 0.0002 & -3767 & -0.00196 & -0.00015 & IBVS No. 6010 \\
\hline 2455479.4217 & & $\mathrm{~s}$ & 0.0007 & -3680.5 & -0.00204 & -0.00016 & IBVS No. 5984 \\
\hline 2455479.4271 & & $\mathrm{~s}$ & 0.0007 & -3680.5 & 0.00336 & 0.00524 & IBVS No. 5984 \\
\hline 2455479.6192 & & $\mathrm{p}$ & 0.0012 & -3680 & -0.00066 & 0.00122 & IBVS No. 5984 \\
\hline 2455479.6192 & & $\mathrm{p}$ & 0.0012 & -3680 & -0.00066 & 0.00122 & IBVS No. 5984 \\
\hline 2455492.16933 & $R$ & $\mathrm{p}$ & 0.00080 & -3648 & -0.00226 & -0.00036 & This paper \\
\hline 2455501.19018 & $R$ & $\mathrm{p}$ & 0.00025 & -3625 & -0.00296 & -0.00104 & This paper \\
\hline 2455535.11975 & $R$ & $\mathrm{~s}$ & 0.00063 & -3538.5 & -0.00227 & -0.00030 & This paper \\
\hline 2455816.15762 & $R$ & $\mathrm{p}$ & 0.00050 & -2822 & -0.00537 & -0.00283 & This paper \\
\hline 2455839.3042 & Ir & $\mathrm{p}$ & 0.0023 & -2763 & -0.00103 & 0.00100 & IBVS No. 6026 \\
\hline 2455839.4988 & Ir & $\mathrm{s}$ & 0.0033 & -2762.5 & -0.00255 & -0.00052 & IBVS No. 6026 \\
\hline 2455849.3067 & Ir & $\mathrm{s}$ & 0.0012 & -2737.5 & -0.00069 & 0.00133 & IBVS No. 6026 \\
\hline 2455849.5019 & Ir & $\mathrm{p}$ & 0.0019 & -2737 & -0.00161 & 0.00041 & IBVS No. 6026 \\
\hline 2455858.7190 & & $\mathrm{~s}$ & 0.0004 & -2713.5 & -0.00218 & -0.00017 & IBVS No. 6011 \\
\hline 2455876.17208 & $R$ & $\mathrm{p}$ & 0.00047 & -2669 & -0.00385 & -0.00186 & This paper \\
\hline 2456614.3740 & & $\mathrm{p}$ & 0.0002 & -787 & -0.00026 & 0.00025 & IBVS No. 6167 \\
\hline 2456923.06759 & $V R I$ & $\mathrm{p}$ & 0.00015 & 0 & -0.00066 & -0.00086 & This paper \\
\hline 2456934.4433 & $I$ & $\mathrm{p}$ & 0.0022 & 29 & 0.00005 & -0.00018 & IBVS No. 6152 \\
\hline 2456934.6401 & $I$ & $\mathrm{~s}$ & 0.0002 & 29.5 & 0.00073 & 0.00050 & IBVS No. 6152 \\
\hline 2456936.2078 & $V R I$ & $\mathrm{~s}$ & 0.0011 & 33.5 & -0.00056 & -0.00079 & This paper \\
\hline 2456936.9970 & $V R I$ & $\mathrm{~s}$ & 0.0012 & 35.5 & 0.00415 & 0.00391 & This paper \\
\hline 2456949.3483 & $I$ & $\mathrm{p}$ & 0.0007 & 67 & -0.00012 & -0.00039 & IBVS No. 6152 \\
\hline 2456949.5454 & $I$ & $\mathrm{~s}$ & 0.0023 & 67.5 & 0.00085 & 0.00059 & IBVS No. 6152 \\
\hline
\end{tabular}


Table 2-Continued

\begin{tabular}{cccrrrrr}
\hline \hline JD (Hel.) & Filter & Min. & Error (d) & Epoch & $(O-C)(\mathrm{d})$ & Residuals (d) & Reference \\
& & & & & & \\
\hline & & & & & & & \\
2457131.5455 & $I$ & $\mathrm{~s}$ & 0.0021 & 531.5 & 0.00094 & 0.00029 & IBVS No. 6196 \\
2457357.2812 & $U I$ & $\mathrm{p}$ & 0.0013 & 1107 & 0.00171 & 0.00061 & IBVS No. 6196 \\
2457657.3452 & & $\mathrm{p}$ & 0.0002 & 1872 & 0.00105 & -0.00054 & OEJV No. 0179 \\
2457657.5416 & & $\mathrm{~s}$ & 0.0002 & 1872.5 & 0.00134 & -0.00025 & OEJV No. 0179 \\
2458018.405 & & $\mathrm{~s}$ & 0.002 & 2792.5 & 0.00261 & 0.00061 & IBVS No. 6244 \\
\hline
\end{tabular}

Table 3: Obital elements of V344 Lac.

\begin{tabular}{ll}
\hline Parameter & Value \\
\hline$T_{0}[\mathrm{cor}](\mathrm{HJD})$ & $2456923.0683(8)$ \\
$P_{0}[\mathrm{cor}](\mathrm{d})$ & $0.39224146(14)$ \\
$\beta\left(\mathrm{d} \mathrm{yr}^{-1}\right)$ & $(1.9 \pm 10.4) \times 10^{-9}$ \\
$A_{3}(\mathrm{~d})$ & $0.0020(2)$ \\
$a_{12} \sin i^{\prime}(\mathrm{au})$ & $0.35 \pm 0.05$ \\
$e$ & $0.38 \pm 0.16$ \\
$\omega(\mathrm{deg})$ & $224 \pm 22$ \\
$P_{3}(\mathrm{yr})$ & $12.4 \pm 0.5$ \\
$H_{p}(\mathrm{HJD})$ & $2459879.0 \pm 902.1$ \\
$f(M)\left(M_{\odot}\right)$ & $(2.788 \pm 0.051) \times 10^{-4}$ \\
$\Delta P / P=2 \pi A_{3} / P_{3}$ & $(2.775 \pm 0.389) \times 10^{-6}$ \\
\hline
\end{tabular}


Table 4: Photometric solutions for V344 Lac.

\begin{tabular}{|c|c|c|c|c|}
\hline Parameters & $\begin{array}{c}\text { Photometric elements } \\
\text { without } l_{3}\end{array}$ & uncertainties & $\begin{array}{c}\text { Photometric elements } \\
\text { with } l_{3}\end{array}$ & uncertainties \\
\hline$g_{1}=g_{2}$ & 0.32 & fixed & 0.32 & fixed \\
\hline$A_{1}=A_{2}$ & 0.50 & fixed & 0.50 & fixed \\
\hline$x_{1 \text { bolo }}, x_{2 \text { bolo }}, y_{1 \text { bolo }}, y_{2 b o l o}$ & $0.136,0.147,0.584,0.572$ & fixed & $0.136,0.144,0.584,0.575$ & fixed \\
\hline$x_{1 V}, x_{2 V}, y_{1 V}, y_{2 V}$ & $0.139,0.161,0.673,0.658$ & fixed & $0.139,0.155,0.673,0.663$ & fixed \\
\hline$x_{1 R}, x_{2 R}, y_{1 R}, y_{2 R}$ & $0.028,0.046,0.695,0.684$ & fixed & $0.028,0.041,0.695,0.687$ & fixed \\
\hline$x_{1 I}, x_{2 I}, y_{1 I}, y_{2 I}$ & $-0.038,-0.022,0.665,0.656$ & fixed & $-0.038,-0.026,0.665,0.659$ & fixed \\
\hline Phaseshift & 0.0011 & \pm 0.0002 & 0.0011 & \pm 0.0002 \\
\hline$T_{1}(\mathrm{~K})$ & 6312 & $\pm 54 \mathrm{~K}$ & 6312 & $\pm 54 \mathrm{~K}$ \\
\hline$T_{2}$ & $6166 \mathrm{~K}$ & $\pm 64 \mathrm{~K}$ & $6201 \mathrm{~K}$ & $\pm 65 \mathrm{~K}$ \\
\hline$q=M_{2} / M_{1}$ & 0.580 & \pm 0.003 & 0.387 & \pm 0.003 \\
\hline$i\left(^{\circ}\right)$ & 67.0 & \pm 0.1 & 70.3 & \pm 0.7 \\
\hline$\Omega_{1}=\Omega_{2}$ & 2.9371 & \pm 0.0054 & 2.5457 & \pm 0.0070 \\
\hline$\Omega_{\text {in }}$ & 3.0258 & - & 2.6512 & - \\
\hline$\Omega_{\text {out }}$ & 2.6853 & - & 2.4145 & - \\
\hline$L_{1} /\left(L_{1}+L_{2}\right)(V)$ & 0.6408 & \pm 0.0028 & 0.7091 & \pm 0.0221 \\
\hline$L_{1} /\left(L_{1}+L_{2}\right)(R)$ & 0.6365 & \pm 0.0024 & 0.7062 & \pm 0.0214 \\
\hline$L_{1} /\left(L_{1}+L_{2}\right)(I)$ & 0.6331 & \pm 0.0021 & 0.7041 & \pm 0.0210 \\
\hline$L_{3} /\left(L_{1}+L_{2}+L_{3}\right)(V)$ & - & - & 0.1054 & \pm 0.0272 \\
\hline$L_{3} /\left(L_{1}+L_{2}+L_{3}\right)(R)$ & - & - & 0.1014 & \pm 0.0266 \\
\hline$L_{3} /\left(L_{1}+L_{2}+L_{3}\right)(I)$ & - & - & 0.0876 & \pm 0.0265 \\
\hline$r_{1}($ pole $)$ & $0.4163 \pm 0.0010$ & $0.4558 \pm 0.0015$ & & \\
\hline$r_{1}($ side $)$ & $0.4441 \pm 0.0013$ & $0.4925 \pm 0.0021$ & & \\
\hline$r_{1}(b a c k)$ & $0.4794 \pm 0.0019$ & $0.5276 \pm 0.0029$ & & \\
\hline$r_{2}($ pole $)$ & $0.3263 \pm 0.0012$ & $0.3018 \pm 0.0021$ & & \\
\hline$r_{2}($ side $)$ & $0.3434 \pm 0.0015$ & $0.3181 \pm 0.0026$ & & \\
\hline$r_{2}($ back $)$ & $0.3859 \pm 0.0027$ & $0.3707 \pm 0.0057$ & & \\
\hline$f$ & $26.0 \%$ & $\pm 1.6 \%$ & $44.6 \%$ & $\pm 3.0 \%$ \\
\hline$\sum(O-C)_{i}^{2}$ & 0.037263 & & 0.037081 & \\
\hline
\end{tabular}



Fig. 1.- Observed and theoretical light curves in VRI passbands of V344 Lac. 
Table 5: Some contact binaries with cyclic variation of their obital period.

\begin{tabular}{|c|c|c|c|c|c|c|c|c|c|c|c|c|c|}
\hline Name & $\begin{array}{l}P \\
\text { (d) } \\
\end{array}$ & $\begin{array}{l}q \\
\left(M_{2} / M_{1}\right)\end{array}$ & $\begin{array}{l}M_{1} \\
\left(M_{\odot}\right)\end{array}$ & $\begin{array}{l}T_{1} \\
(\mathrm{~K}) \\
\end{array}$ & $\begin{array}{l}T_{2} \\
(\mathrm{~K}) \\
\end{array}$ & $\begin{array}{l}P_{3} \\
(\mathrm{yr})\end{array}$ & $\begin{array}{l}A_{3} \\
\text { (d) } \\
\end{array}$ & $\begin{array}{l}\Delta P / P \\
\left(\times 10^{-6}\right)\end{array}$ & $\begin{array}{l}m_{3} \\
\left(M_{\odot}\right)\end{array}$ & spot & $\begin{array}{l}l_{3} \\
(\%) \\
\end{array}$ & $e$ & reference \\
\hline V776 Cas & 0.440416842 & 0.130 & 1.55 & 7000 & 6920 & 23.7 & 0.0109 & 7.912 & 1.04 & & 14.9 & 0 & (1) \\
\hline V802 Aql & 0.2677087 & 0.144 & 0.92 & 5000 & 5048 & 8.32 & 0.0196 & 40.526 & 1.52 & $\mathrm{y}$ & 0 & 0 & (2) \\
\hline MQ UMa & 0.4760662 & 0.195 & & 6532 & 6224 & 13.6 & 0.0056 & 7.084 & & & 25.1 & 0 & (3) \\
\hline VZ Lib & 0.35825797 & 0.236 & 1.31 & 5446 & 5770 & 2.96 & 0.0039 & 22.666 & 0.52 & $\mathrm{y}$ & 10.4 & 0 & (4) \\
\hline EZ Hya & 0.44974752 & 0.257 & 1.37 & 5721 & 6100 & 30.9 & 0.0185 & 10.299 & 0.51 & & 0 & 0 & (5) \\
\hline DF CVn & 0.3269 & 0.285 & 0.93 & 5520 & 4812 & 17.2 & 0.0070 & 7.001 & 0.23 & $\mathrm{y}$ & 0 & 0 & (6) \\
\hline V1005 Her & 0.278962083 & 0.301 & 0.92 & 5535 & 5560 & 18.1 & 0.0127 & 12.070 & 0.45 & $\mathrm{y}$ & 4.2 & 0 & (7) \\
\hline SS Ari & 0.40598629 & 0.307 & 1.30 & 5488 & 5860 & 37.75 & 0.0112 & 5.104 & 0.278 & $\mathrm{y}$ & 0 & 0 & (8) \\
\hline GN Boo & 0.3016022 & 0.320 & 0.99 & 6250 & 6879 & 9.89 & 0.0042 & 7.306 & 0.21 & & 0 & 0 & (9) \\
\hline $\mathrm{AQ}$ Com & 0.28133036 & 0.350 & 0.95 & 4911 & 5300 & 8.5 & 0.0016 & 3.238 & 0.08 & $\mathrm{y}$ & 0 & 0 & (10) \\
\hline GSC 03526-01995 & 0.29225601 & 0.351 & 0.80 & 4581 & 4830 & 7.39 & 0.0090 & 20.858 & $>0.57$ & $\mathrm{y}$ & 0 & 0 & (11) \\
\hline EP And & 0.4041088 & 0.372 & 1.10 & 6171 & 6250 & 40.89 & 0.0109 & 4.586 & 0.23 & & 9.3 & 0 & (12) \\
\hline GSC $1537-1557$ & 0.31827564 & 0.378 & 0.95 & 5631 & 5740 & 8.1 & 0.0034 & 7.115 & $>0.19$ & $\mathrm{y}$ & 0 & 0 & (13) \\
\hline RT LMi & 0.37491782 & 0.378 & 1.29 & 6400 & 6513 & 46.7 & 0.0049 & 1.805 & & & 0 & 0 & (14) \\
\hline GW Cep & 0.31883088 & 0.386 & 1.11 & 5800 & 6104 & 32.63 & 0.0089 & 4.692 & 0.22 & $\mathrm{y}$ & 0 & 0.076 & (15) \\
\hline V344 Lac & 0.39224141 & 0.387 & 1.16 & 6312 & 6201 & 12.4 & 0.0020 & 2.775 & 0.79 & & 9.81 & 0.38 & (16) \\
\hline V396 Mon & 0.39634132 & 0.392 & 0.92 & 6121 & 6210 & 42.4 & 0.0160 & 6.492 & 0.31 & $\mathrm{y}$ & 0.87 & 0 & (17) \\
\hline TY UMa & 0.35454813 & 0.396 & 1.57 & 6229 & 6250 & 51.7 & 0.0182 & 6.056 & 0.434 & $\mathrm{y}$ & 0 & 0.73 & (18) \\
\hline FV CVn & 0.31536519 & 0.410 & 0.86 & 5150 & 5465 & 17.65 & 0.0184 & 17.934 & $>0.73$ & & 27.7 & 0 & (19) \\
\hline PP Lac & 0.40116138 & 0.435 & 1.18 & 5202 & 5480 & 19.7 & 0.0058 & 5.065 & 0.19 & & 0 & 0 & (20) \\
\hline EQ Tau & 0.3413478 & 0.442 & 1.22 & 5800 & 5740 & 22.7 & 0.0058 & 4.395 & 0.2 & $\mathrm{y}$ & 0 & 0.47 & (21) \\
\hline UZ CMi & 0.55136313 & 0.452 & & 6250 & 6194 & 21.1 & 0.0026 & 2.120 & & & $<1$ & 0 & (22) \\
\hline LU Lac & 0.29880186 & 0.480 & 0.95 & 4899 & 5310 & 51.92 & 0.0125 & 4.142 & 0.21 & & 0 & 0 & (23) \\
\hline CW Cas & 0.31886304 & 0.486 & 0.99 & 4950 & 5390 & 69.9 & 0.0320 & 7.875 & 0.52 & $\mathrm{y}$ & 9.98 & 0.571 & (24) \\
\hline AH Tau & 0.332672375 & 0.505 & 1.04 & 5840 & 5816 & 54.62 & 0.0349 & 10.992 & 0.767 & $\mathrm{y}$ & 0 & 0 & (25) \\
\hline V508 Oph & 0.34479141 & 0.552 & 1.01 & 5980 & 5893 & 24.27 & 0.0036 & 2.552 & 0.1 & $\mathrm{y}$ & 0 & 0 & (26) \\
\hline V1107 Cas & 0.27341123 & 0.556 & 0.70 & 4657 & 5200 & 6.74 & 0.0023 & 5.870 & 0.091 & & 1.83 & 0 & (27) \\
\hline AL Cas & 0.5005555 & 0.610 & 1.23 & 6400 & 6136 & 86.6 & 0.0181 & 3.596 & 0.28 & & 0 & 0 & (28) \\
\hline PY Lyr & 0.38576 & 0.660 & 1.51 & 6980 & 7042 & 52.5 & 0.0395 & 12.943 & 1.15 & $\mathrm{y}$ & 19.7 & 0.138 & (29) \\
\hline PY Vir & 0.31124849 & 0.773 & 0.95 & 4830 & 4702 & 5.22 & 0.0075 & 24.717 & 0.79 & & 0 & 0 & (30) \\
\hline V1044 Her & 0.24064058 & 0.833 & & 4377 & 4575 & 14.1 & 0.0026 & 3.172 & $>0.109$ & $\mathrm{y}$ & 0 & 0.31 & (31) \\
\hline FZ Ori & 0.399986 & 0.886 & 1.17 & 5940 & & 30.1 & 0.0133 & 7.601 & 0.56 & & 0 & 0 & (32) \\
\hline BI Vul & 0.25182367 & 0.966 & & 4474 & 4600 & 10.8 & 0.0057 & 9.079 & & $\mathrm{y}$ & 0 & 0 & (33) \\
\hline DM Del & 0.4198144 & & & & & 51.22 & 0.0218 & 7.322 & 0.12 & & 0 & 0 & (34) \\
\hline V803 Aql & 0.2634262 & & & & & 74.6 & 0.0336 & 7.748 & 0.52 & & 0 & 0 & (29) \\
\hline \multicolumn{14}{|c|}{ 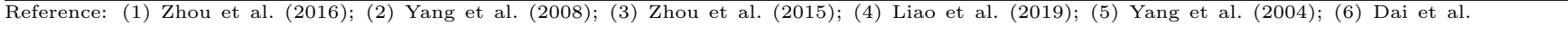 } \\
\hline \multicolumn{14}{|c|}{ 2011; (7) Zhu et al. 2019); (8) Liu et al. 2009); (9) Yang et al. 2013); (10) Liu et al. 2014; ; (11) Liao et al. (2012); (12) Liao et al. (2013); } \\
\hline \multicolumn{14}{|c|}{ (13) Xiang et al. 2015a; (14) Qian et al. 2008; (15) Lee et al. 2010; (16) The present paper; (17) Liu et al. 2011; (18) Li et al. 2015; (19) } \\
\hline \multicolumn{14}{|c|}{ Liao \& Sarotsakulchai 2019); (20) Qian et al. 2005; (21) Li et al. 2014); (22) Qian et al. 2013a); (23) Liao et al. 2014; (24) Wang et al. } \\
\hline \multirow{2}{*}{\multicolumn{14}{|c|}{$\begin{array}{l}\text { 2014); (25) Xiang et al. (2015b; ; (26) Xiang et al. (2015c); (27) Liu et al. (2016); (28) Qian et al. (2014a); (29) Zasche et al. (2009); (30) Zhu et } \\
\text { al. (2013); (31) Lu et al. (2016); (32) Prasad et al. 2014); (33) Qian et al. (2013b); (34) He \& Qian } \sqrt{2010) .}\end{array}$}} \\
\hline & & & & & & & & & & & & & \\
\hline
\end{tabular}






Fig. 2.- Spectrum of V344 Lac. Black solid line is the observed spectrum, while the red solid line is the fitting one. 




Fig. 3.- The $(O-C)$ diagram of V344 Lac was formed by all CCD available measurements. The $(O-C)$ values were computed by using a newly determined linear ephemeris (Eq 1). Full circles refer to the primary times of minima and empty ones to the secondary times of minima. Solid line represents the cyclic fitting (Eq2), while the dashed line is a zero line.

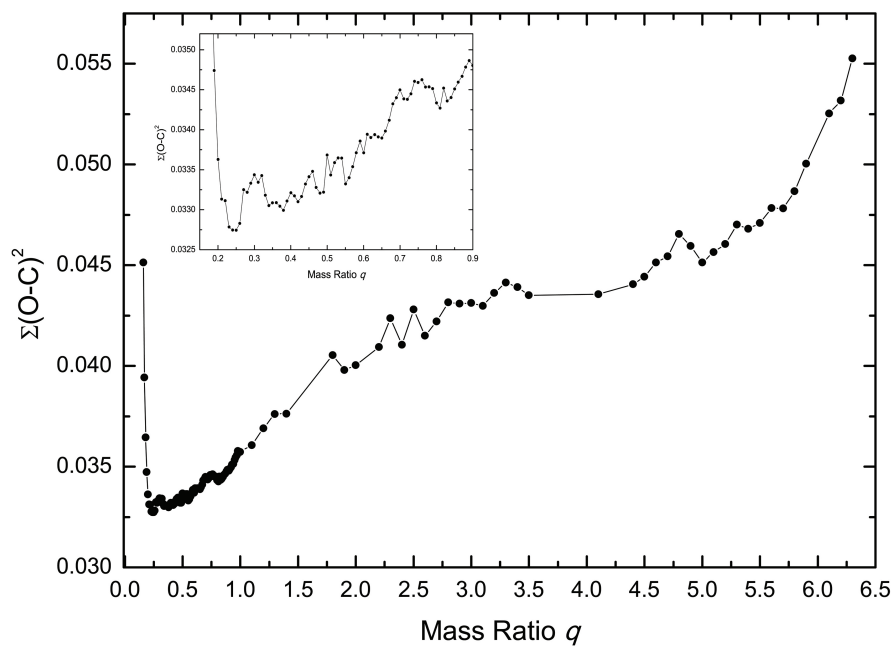

Fig. 4.- The relationship between the mass ratio $q$ and the fitting residuals. 


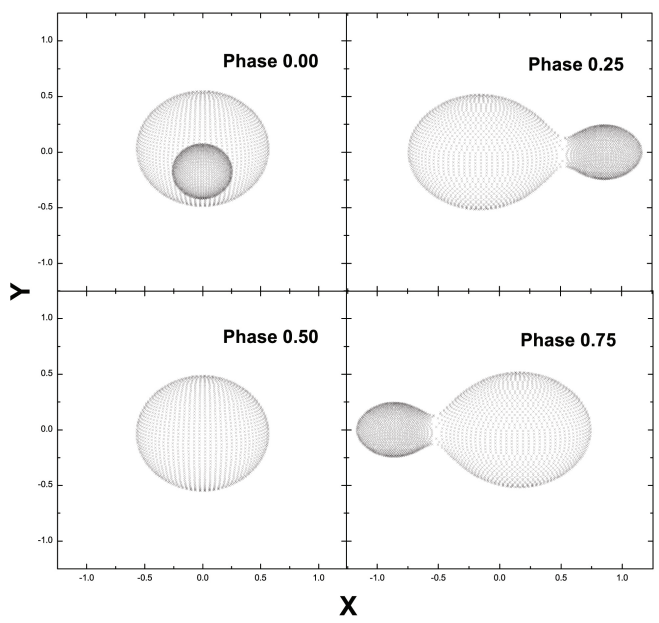

Fig. 5.- Geometric structure of V344 Lac at phase 0.00, 0.25, 0.50 and 0.75.

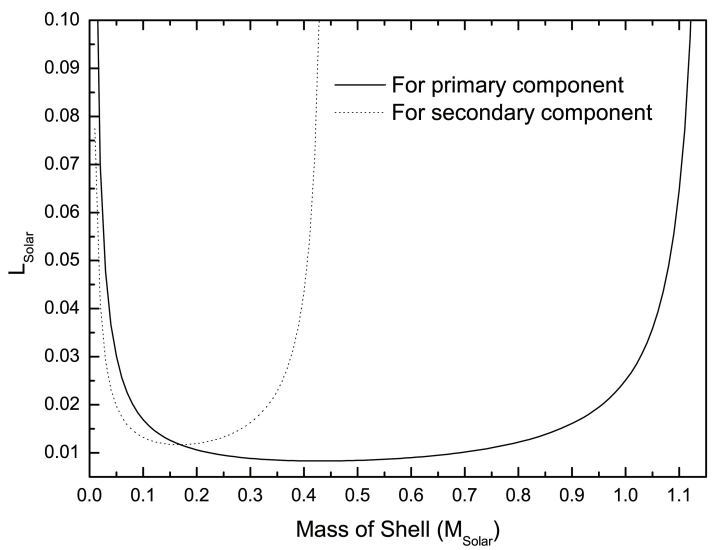

Fig. 6.- Luminosity required to produce the cyclic oscillation in the $(O-C)$ diagram using the Applegate's mechanism. The solid line denotes the required luminosity for the primary component (more massive one), while the dashed line denotes that for the secondary. 